\title{
New Target Results from the FNAL Antiproton Source
}

\author{
S. O'Day, F. Bieniosek, K. Anderson \\ Fermilab,P.O. Box 500,Batavia,IL 60510
}

\section{Abstract}

Nickel and compressed rhenium powder targets have been installed in the FNAL antiproton source target station. Ni was chosen for its high melting point energy and resistance to stress wave fractures. As well, compressed powdered rhenium segments were constrained by a thinwall Ti jacket to insure resistance to stress fractures. The $\bar{p}$ yield of these new targets is compared with that of copperthe previous standard production target. The target depletion characteristics of nickel and rhenium for a beam intensity of $1.6 \times 10^{12}$ protons per pulse are also presented.

\section{Introduction}

The requirements placed on the antiproton production target at Fermilab become more stringent as the linac upgrade and Main Injector projects come to fruition. To understand how to meet the yield and durability requirements that these projects will impose, a variety of targets have been considered. The targets which were installed for production and study in February of 1992 were selected carefully based on their material properties.

The properties which are the most interesting are yield strength, melting point and shock wave limit energies and the Gruneisen parameter (which relates the differential pressure to energy changes). Ideally, one would like to have a target material with a high yield strength and shock wave limit energy, but a small Gruneisen parameter to minimize local pressure gradients. The melting point energy should be as high as is necessary to eliminate or at least minimize localized melting at the beam spot for a single beam pulse. Melting will reduce the density thus diminishing the yield. Data for various target materials are given in Table 1.

Calculating the expected yields for various targets is difficult. Efforts in the past have produced results which are only within a factor of 2 of measured yields ${ }^{1}$. The antiproton yield is only weakly dependent on the absorption length(table 1) complicating even relative yield estimates. As more data is collected, modeling efforts will show improved results. Qualitative statements can however be made regarding the material dependence of $\bar{p}$ production. While targets with shorter absorption lengths experience a greater number of interactions with $120 \mathrm{GeV}$ protons, the yield gain is tempered by the greater fraction of produced $\overline{p s}$ which are re-absorbed in the target. Also, primaries from proton-nucleon inelastic scattering and secondaries

\footnotetext{
* Operated by the Universities Research Association un-
} der contract with the U.S. Department of Energy
Table 1

Target Material Properties

\begin{tabular}{|c|c|c|c|c|c|c|}
\hline $\mathbf{T}_{\mathbf{B}^{\ddagger}}$ & Iridium & Rhenium & Tungsten & Nickel & Copper & Lon \\
\hline$A ! / P$ & .255 & .271 & .295 & .437 & .446 & .486 \\
\hline Melting Pt. $E\left(J / g^{m}\right)$ & 458 & 614 & 634 & 1044 & 613 & 1030 \\
\hline Yield Stzength $(\mathrm{Pa})$ & 160000 & 270000 & 500000 & 230000 & 72000 & 134000 \\
\hline$\rho \Gamma\left(P_{a} K_{g} / S\right)$ & 80600 & 66000 & 31000 & 15800 & 17200 & 15800 \\
\hline Shack Wave Limit $(\mathrm{g} / \mathrm{gm})$ & & & 200 & & 600 & \\
\hline
\end{tabular}

from primary hadron-nucleon interactions both contribute to the $\bar{p}$ yield. The admixture of these processes is both geometry and material dependent. Figure 1 shows the target stack geometry which was used for this experiment.

In $\bar{p}$ production running, a $120 \mathrm{GeV}$ beam of $1.6 \times 10^{12}$ protons per pulse ( 80 full bunches per pulse) impinges on a selected target. A lithium lens approximately $20 \mathrm{~cm}$ downstream collects and focuses the $8 \mathrm{GeV}$ secondaries. These secondaries are then kicked horizontally 3 degrees by a pulsed dipole into the aperture of the AP2 beamline and transported to the Debuncher storage ring.

During optimum running conditions, there may be as many as $2.1 \times 10^{12}$ protons per pulse on target and the operating gradient of the lithium collection lens may be as high as $890 \mathrm{~T} / \mathrm{m}$. Under such conditions, the measured absolute yield in to the Debuncher was measured to be $1.9 \times$ $10^{-5} \bar{p} / p$ or $4.0 \mu \mathrm{A}$ of current per pulse.

\section{Measurements}

\section{A. Yield Measurements}

The yield measurements were made in the Debuncher using both a dynamic signal analyzer(dsa) and a d.c. beam current transformer(dect). The signal analyzer does an FFT of the longitudinal Schottky power spectrum and integrates it over a specified bandwidth. The signal analyzer is calibrated to the dcct and is accurate to a few percent. A summary of the results may be found in Table 2. The yield is normalized to the proton intensity on target as given by the toroid in the $120 \mathrm{GeV}$ beamline(AP1) closest 


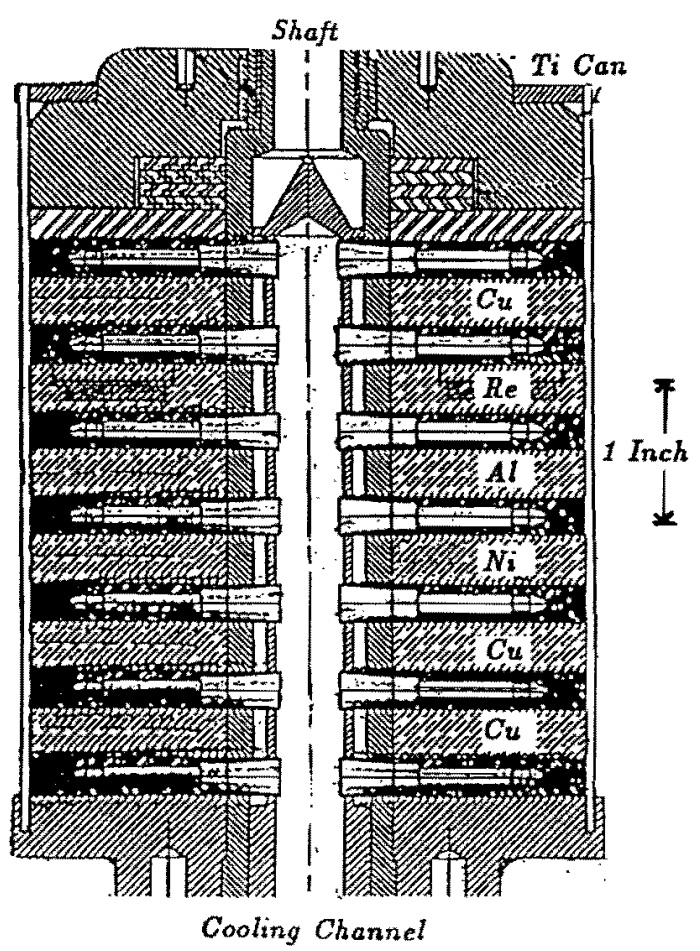

Figure 1.Target Stack. Note that the target stack has vertical motion for changing targets, horizontal motion perpendicular to the beam for changing the length of target which the beam intersects and motion along the beam so that the lens focal point may be varied. The beam direction is into the page.

Table 2

Yield Data Normalized to Ni

\begin{tabular}{|c|c|c|}
\hline Material & DSA/AP1 Toroid & DCCT/AP1 Toroid \\
\hline Nickel & 1.000 & 1.000 \\
\hline Copper & 0.979 & 0.997 \\
\hline Rhenium & 0.994 & 0.998 \\
\hline
\end{tabular}

to the target. These data show no statistically significant differences between the $\bar{p}$ yields of the three targets.

\section{B. Target Edge Yield Study}

The $\bar{p}$ yield was measured when the beam hit near the interface between copper and aluminum. Copper is much denser than aluminum. The study attempted to find out whether or not the yield was enhanced by allowing roughly a third of the solid angle of primarily produced $\bar{p}$ s to escape through a lighter material thus lowering the probability of

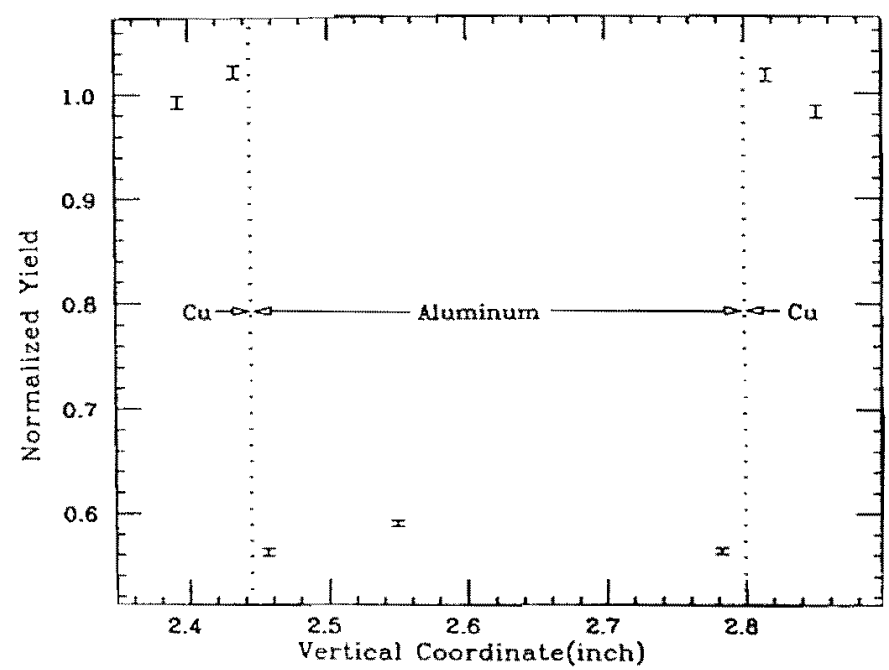

Figure 2. Yield enhancement at the target vertical edge.

$\bar{p}$ absorption. At the same time however, the probability of producing $\bar{p}$ s from energetic pions is reduced because these will also escape through the aluminum with a much lower probability of inelestic interaction.

To compare with theoretical predictions which ignore secondary $\bar{p}$ production, the collection lens was focused on the upstream end of the target. Here, a larger fraction of the $\bar{p}$ s produced would be primary hadrons. Figure 2 shows that edge enhancement on the order of $4-5 \%$ is observed. The overall yield is however reduced by $30 \%$. Future studies will include a $1 \mathrm{~mm}$ diameter wire target which should further enhance this effect and possibly increase the overall $\overline{\boldsymbol{p}}$ yield.

\section{Target Density Depletion Study}

A large energy deposition on target is expected to locally melt target materials. This will result in a diminished hadron yield from those bunches of protons which arrive after this melting has occurred. Since rhenium has a calculated melting point energy below our energy deposition level, we performed a study to demonstrate density depletion using this target.

The FNAL Main Ring provided a beam with an intensity of $1.6 \times 10^{12}$ protons per pulse and a transverse width $(\sigma)$ of $.14 \mathrm{~mm}$ on target. Using a resistive wall monitor in the Debuncher, the bunch structure of the beam during the first turn was measured. As well, a similar wall monitor was used to measure the incoming proton bunch structure. By integrating the individual bunches, the charge associated with each bunch was determined. Comparing the production bunch charge with the corresponding proton bunch charge provides a measure of the 
Bunch Charge vs. Bunch Number(Rhenium Target)

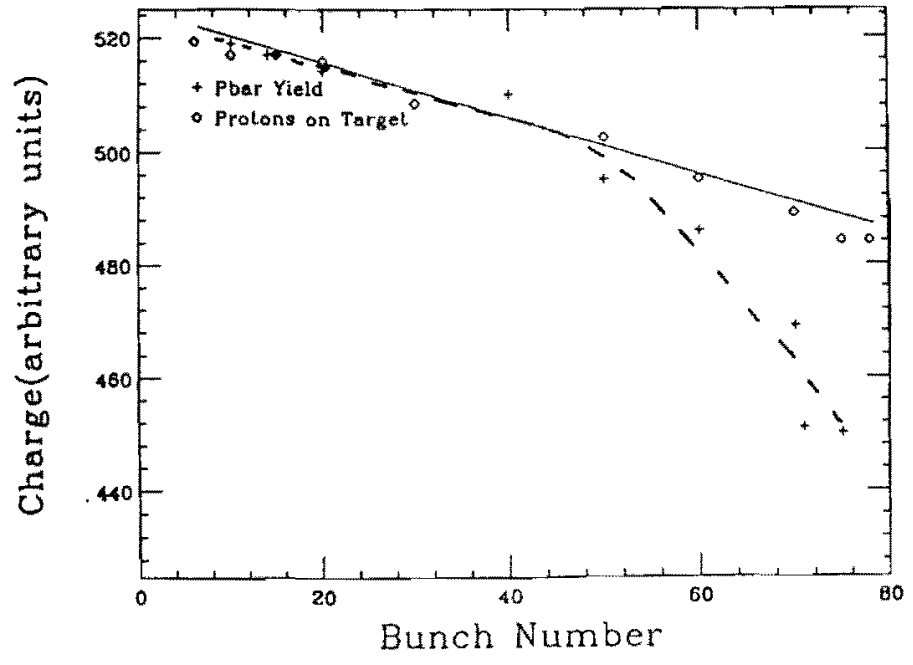

Figure 3. Yield vs. Bunch Length

yield. The charge in arbitrary units for protons on target and $\beta=1$ particles in the Debuncher as a function of bnnch number is given in figure 3 . The yield from the last $25 \%$ of the bunches shows an obvious deficit which increases with bunch number. By the eightieth bunch, the yield is down $8 \% \pm 4 \%$ as compared with the first.

Also interesting is the number of bunches which hit the target before the yield began to drop. The yield began to drop after $45 \pm 5$ bunches or after a Casim calculated energy deposition of $600 \pm 65 \mathrm{~J} / \mathrm{gm}$. This is consistent with the melting point energy for rhenium from table 1 of 614 $\mathrm{J} / \mathrm{gm}$. A more sophisticated model assuming the relationship $d \rho / d T=-.0001 \rho$ for liquid rhenium and a $25 \%$ beam absorption rate in the central beam region predicts that $\Delta \rho / \rho=-21 \%$. Since the melting point of rhenium is $3180^{\circ} \mathrm{C}$ and a density drop of this magnitude correspond$s$ to a temperature of $5280^{\circ} \mathrm{C}$, this implies that the the melting began after $60 \%$ of the pulse hit the target. This is once again consistent with the data.

Analysis of the target station airborne radiation monitor filter revealed the presence of radionuclides(largely iodine, with smaller amounts of tellurium, sodium and potassium) in the airborne radiation monitor ${ }^{2}$. The total release was quite small( about $5 \mathrm{mCi}$ ). This release is a by-product of the spallation reactions between the $120 \mathrm{GeV}$ protons and the target nuclei ${ }^{3}$ and the subsequent nuclear fission. These reactions produce noble gases (xenon in this case) and result in void formation,swelling and embrittlement of solid target materials. We hypothesize that the xenon, normally trapped in the solid metal target, was released as the target melted and escaped through the voids in the pressed-powder target material and the seams of the titanium can. Radioactive xenon nuclei would then have decayed to the daughter products observed(iodine and tellurium). A similar, although much smaller, release occurred with an intensity of $1.2 \times 10^{12}$ protons per pulse.

It should be noted that no target depletion effects were observed for $\mathrm{Ni}$ ( the standard production target at this time). This is what one would expect since the energy deposition in $\mathrm{Ni}$ for an intensity of $1.6 \times 10^{12}$ protons per pulse is $615 \mathrm{~J} / \mathrm{gm}$ while the melting point energy is $1100 \mathrm{~J} / \mathrm{gm}$.

\section{Conclusions}

From the measurements made, one can conclude that the yields from quite different materials- $\mathrm{Ni}$ and $\mathrm{Cu}$ as compared with Re, are similar. Also, the calculations and measurements made were in agreement on the issue of target density depletion. The yield enhancement measuremen$t$ leads naturally to a more sophisticated target geometry experiment. Specifically, a wire target surrounded by a low $z$ material will be constructed sometime in the future to get more data on the admixture of primary and secondary yield contributions. Further work, both theoretical(Monte Carlo) and experimental is planned to improve the understanding of $\bar{p}$ production. This work will lead to the optimization of future target and collection lens designs.

\section{References}

1. C. Hojvat, A. Van Ginneken, "Calculation of Antiproton Yields for the Fermilab Antiproton Source",Nucl.Instrum. Meth.206:67,1983.

2. A. Leveling, AD Radiation Safety, FNAL. Private Communication. (January 1993.)

3. T.W. Eaton, C.D. Johnson, E. Jones,CERN/PS/86815,1986 . 